\title{
Intracellular manipulation of chromatin using magnetic nanoparticles
}

\author{
Johannes S. Kanger ${ }^{1 *}$, Vinod Subramaniam ${ }^{1} \&$ Roel van Driel $^{2}$ \\ ${ }^{1}$ Biophysical Engineering, Faculty of Science and Technology, Institute for Biomedical Technology (BMTI), \\ University of Twente, PO Box 217, 7500 AE, Enschede, The Netherlands; Tel: +31-53-4893726; Fax: +31-53- \\ 4891105; E-mail: j.s.kanger@utwente.nl; ${ }^{2}$ Swammerdam Institute for Life Sciences, University of Amsterdam, \\ Amsterdam, The Netherlands \\ * Correspondence
}

Key words: cell nucleus, chromatin structure, magnetic tweezers, nanoparticles

\begin{abstract}
\section{Abbreviations}

AFM atomic force microscopy

2D two-dimensional

3D three-dimensional

GFP green fluorescent protein

MT magnetic tweezers

$\mathrm{nN}$ nanonewton $\left(10^{-9} \mathrm{~N}\right)$

OT optical tweezers

$\mathrm{pN}$ piconewton $\left(10^{-12} \mathrm{~N}\right)$

PTM particle tracking microrheology

VSM vibrating sample magnetometer
\end{abstract}

Magnetic tweezers are widely used for manipulating small magnetic beads inside the cell cytoplasm in order to gain insight into the structural and mechanical properties of the cytoskeleton. Here we discuss the use of magnetic tweezers for the study of nuclear architecture and the mechanical properties of chromatin in living cells. A custom-built, dedicated micro magnetic tweezer set-up is described. We review progress that has been made in applying this technology for the study of chromatin structure and discuss its prospects for the in situ analysis of nuclear architecture and chromatin function.

\section{Introduction}

Understanding of the importance of nuclear architecture and its relation to the physical properties of chromatin has been the stimulus for many experiments aimed at the characterization of the mechanical properties of chromatin structure. Several techniques are applied to study the mechanical properties of chromatin. Optical tweezer studies on reconstituted single chromatin fibers have shed light on the forces that are required to probe the $30 \mathrm{~nm}$ fiber structure and the bead-on-a-string $10 \mathrm{~nm}$ fiber of chromatin (Cui \& Bustamante 2000, Bennink et al. 2001). The mechanical properties of chromatin on a higher structural level, that of metaphase chromosomes, were studied using micropipettes that act as force probes (Marko \& Poirier 2003). This method, however, is limited to isolated chromosomes. More recent studies have been done on nuclear structure in living cells. For example, light microscopy has been used to measure diffusion of small solutes and proteins (Phair \& Mistelli 2000). Particle tracking microrheology (Tseng et al. 2004) and magnetic tweezers (MT) (de Vries et al. 2007) were successfully applied to the nucleus of a living cell. Isolated individual nuclei have been studied using micropipette aspiration (Lammerding et al. 2007), microplate manipulation (Thoumine \& Ott 1997), and atomic force microscopy (Dahl et al. 2005), among other approaches.

These methods have been developed to study the physical properties of chromatin. However, no 
experiments have been aimed at the active manipulation of chromatin structure and, in this way, at the analysis of the relevance of chromatin structure for genome function, e.g. gene expression. Techniques such as atomic force microscopy, optical tweezers and magnetic tweezers, which exploit the unique capabilities provided by nano- and micro-technologies and advanced optics, are good candidates for manipulation of chromatin structure in living cells. We have focused on the development of a magnetic tweezer method especially for this purpose.

In this paper we review different methods for intracellular manipulation, discuss their applicability for studying nuclear structure, give a detailed description of the development of a dedicated magnetic tweezers, describe magnetic tweezer experiments on nuclear structure, and finally discuss future application of this method for the study of chromatin structure-function relationships.

\section{Intracellular manipulation}

In this section we review the most important techniques for intracellular manipulation and discuss the applicability for intracellular chromatin manipulation. There are basically two ways to manipulate structures inside a cell. The first method relies on the introduction into cells of chemical compounds that modify cellular structures (e.g. cytochalasin D, which inhibits actin polymerization). Here, we limit ourselves to the second method: mechanical manipulation. In this method, a force is exerted on an intracellular structure, or on a part thereof, to either deform it and/or reposition it within the cell. The three most viable techniques for mechanical manipulation are optical tweezers, atomic force microscopy (or micropipettes) and magnetic tweezers, and these are depicted schematically in Figure 1.

Optical tweezers (OT) use a strongly focused laser beam to allow $3 \mathrm{D}$ positioning of microparticles by application of radiation force. Usually, the microparticles (e.g. polystyrene beads) are connected to the intracellular structure of interest. One example is the manipulation in the cell of phagocytosed polystyrene beads (Caspi et al. 2002). Alternatively, organelles can be manipulated directly by OT, without the introduction of microparticles (Shelby et al. 2005). This mode of operation makes OT a truly non-invasive technique, but is also one of the main drawbacks of OT for intracellular manipulation. All optically dense structures in the cell in close vicinity of the focus of the optical trap will be pulled towards it thus making the OT not very specific. The forces that can be applied are rather low $(1-10 \mathrm{pN})$ unless very high laser powers are used, but this increases the risk of laser-induced cell damage. Recent developments have shown that this photodamage can be minimized by using specially shaped OTs (Jeffries et al. 2007).

Magnetic tweezers (MT) were first applied to cells for the measurement of elasticity of the cytoplasm and its viscosity (Crick \& Hughes 1950, Bausch et al. 1999). Magnetic beads are introduced into the cell and an externally generated magnetic field gradient exerts a magnetic force on the bead. Because cells do not normally contain magnetic structures, the force is very specifically applied to the internalized magnetic (a) OT

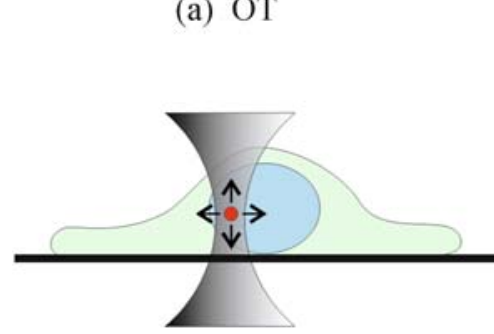

(b) MT

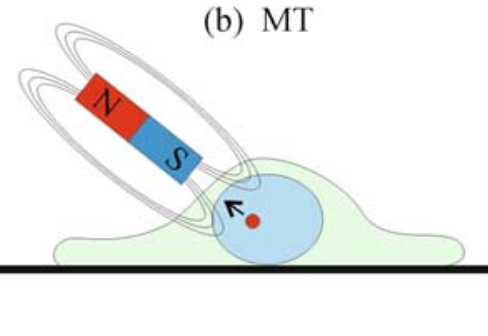

(c) AFM

Figure 1. Experimental schemes for mechanical manipulation of chromosomes in live cells. External forces can be applied on the internal nuclear structures either directly or by means of microinjected beads. This could in principle be used to rearrange chromatin distribution in the nucleus of living cells. The three main methods for applying the external force are (a) optical tweezers (OT) that use a highly focused laser beam to exert forces on a microinjected polystyrene bead, (b) magnetic tweezers (MT) that are used to exert a magnetic force on a magnetic bead, and (c) atomic force microscopy (AFM) or microneedle manipulation that can be used to directly interact with nuclear structure. 
particle. Furthermore, magnetic fields do not interfere with the cell function. Magnetic tweezers are also used for manipulation of internal cellular structures such as phagosomes (Feneberg et al. 2001). Forces that can be applied range from a few piconewtons (Strick et al. 1996) to several nanonewtons (Bausch et al. 1998). Although most MT systems described in the literature apply forces in one direction only, there are a growing number of designs that allow 2D (de Vries et al. 2007) and even 3D (Fisher et al. 2005) manipulation.

Atomic force microscopy (AFM) and the closely related microneedle technique have also been used to apply forces to structures inside a cell. Skibbens and colleagues (Skibbens \& Salmon 1997) used microneedles to apply mechanical forces to the spindle in pre-anaphase newt epithelial cells. To this end, glass microneedles are pushed through the membrane into the nucleus by means of a micromanipulator. Recently, a modified AFM using nanoneedles was developed (Obataya et al. 2005a). These nanoneedles can be used to penetrate the cell membrane to manipulate internal cellular structures (Obataya et al. 2005b). AFM allows careful positioning of the nanoneedle and simultaneous measurement of the applied forces.

Of the technologies described above, only MT (de Vries et al. 2007) and AFM (Skibbens \& Salmon 1997) have been used so far to probe nuclear structure. Owing to a lack of precise knowledge of the mechanical and structural properties of nuclear structure, it is difficult to assess the potential of the different techniques for intracellular chromatin manipulation. Clearly AFM allows much higher forces to be exerted (many nanonewtons) than MT (piconewtons to nanonewtons). This makes AFM more suitable for manipulating large nuclear structures, whereas MT are most likely limited to the rearrangement of smaller structures or rearrangements over smaller length scales. OT are limited to the application of even lower forces. Both MT and OT are attractive techniques as they can be regarded as minimally invasive: once a force probe (either a magnetic or non-magnetic bead) is inserted into the cell nucleus, the manipulation of the probe is performed without "touching" the cell. In general, we believe that AFM, MT and OT are all potential candidates for successful intracellular manipulation. Of all three techniques, MT is especially promising as the MT approach produces minimal cell damage, is highly selective, and allows moderate forces to be exerted. This paper focuses on nanomanipulation of chromatin using MT.

\section{Micro magnetic tweezers}

In this section we outline some basic concepts in magnetic tweezer design and describe in more detail our custom-designed magnetic tweezer set-up. The aim of manipulating chromatin inside the nucleus of a living cell requires (i) sufficiently high forces to be able to disturb chromatin structure, and (ii) small bead sizes ( $1 \mu \mathrm{m}$ or smaller). The translational magnetic force $\boldsymbol{F}_{\mathbf{m}}$ that is exerted on a magnetic particle in the presence of an external magnetic field $\boldsymbol{H}$ with flux density $\boldsymbol{B}$ depends both on the gradient in the magnetic flux density and on the magnetic moment $\boldsymbol{m}$ of the particle: $\boldsymbol{F}_{\mathbf{m}}=\nabla(\boldsymbol{m} \cdot \boldsymbol{B})$. Typically superparamagnetic beads are used as they do not have a magnetic moment in the absence of an external magnetic field. In practice, nanonewton forces are achievable for micrometer-sized superparamagnetic particles. However, for smaller particles the force drops rapidly as the magnetic moment $\boldsymbol{m}$ of the particle scales with the volume of the particle ( $\boldsymbol{m} \sim r^{3}$, with $r$ the radius of the particle). Therefore, the challenge is to generate magnetic fields with very large gradients in the magnetic flux density. Usually magnetic fields are generated using a solenoid (electric coil) with a magnetic core. The use of electromagnets has an advantage over the use of permanent magnets, as they allow for easy control of the amplitude of the magnetic field and thus that of the exerted magnetic force. High gradients in the magnetic flux density are achieved by using a magnetic yoke that guides the generated magnetic flux to a magnetic pole with a sharp tip. Typically, the maximum gradient that can be achieved scales with the dimensions of the tip $\beta$ and with the distance from the tip $R$ as $\nabla \boldsymbol{B} \sim \beta^{-1} \sim R^{-1}$ (de Vries et al. 2005). Therefore, small tip radii should be used and the tip should be positioned close to the bead. Typical optimized tip dimensions to address single cells are of the order of $10 \mu \mathrm{m}$ (de Vries et al. 2005).

The general design of our custom-built micro magnetic tweezers is shown in Figure 2. It consists of an inverted microscope (here a IMT-2 Olympus, Tokyo, Japan) equipped with a video camera and a video recording device, a custom-designed magnetic 

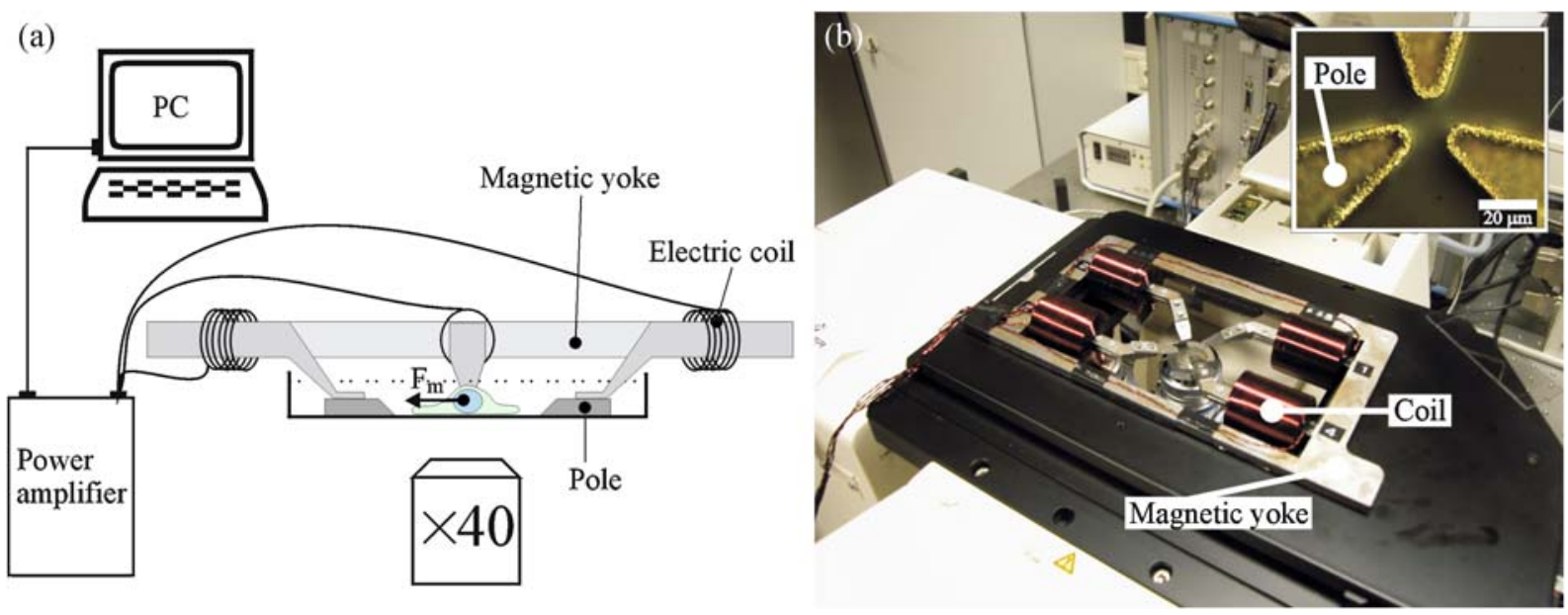

Figure 2. (a) Schematic representation of the micro magnetic tweezer set-up used in our laboratory. The current in the coils is computer controlled. $F_{\mathrm{m}}$ denotes the magnetic force exerted on the particle by the magnetic tweezers. (b) Photograph of the magnetic tweezers assembled on a commercial microscope. Visible are four electric coils of which only three are used in the 3-pole magnetic tweezer configuration. The inset shows a light-microscopy image of the cobalt 3-pole assembly.

yoke with a number of electric coils (1000 windings, maximum current $0.75 \mathrm{~A}$ ), a computer that controls the current through the coils, and microfabricated pole structures. In order to achieve high gradients in the magnetic flux density, it is important to carefully design the configuration (e.g. number, shape and position) of the magnetic poles. The calculation of magnetic field distributions of arbitrary shaped magnetic structures is only possible using numerical calculations. Analysis of different pole geometries using finite-element software (FEMLAB, Comsol, Stockholm, Sweden) resulted in a three-pole design. It is essential to have more than one pole in order to (i) achieve high gradients and (ii) allow changing the direction of the magnetic force (2D manipulation). For example, in a three-pole configuration, in one pole the magnetic field lines point outwards, whereas the other two poles act as a 'sink'. The three poles are arranged on a circle with a radius of $10 \mu \mathrm{m}$, leaving a working area of $20 \mu \mathrm{m} \times 20 \mu \mathrm{m}$, which is large enough to fit a single cell. This pole design allows for real 2D manipulation. An essential feature of this design is that a reasonably constant force is achieved across the central $10 \mu \mathrm{m} \times 10 \mu \mathrm{m}$ of the working area in contrast to, for example, a four-pole design for which the force strongly depends on the position within the central working area. The calculated maximum achievable gradient in the magnetic flux density is $\nabla \boldsymbol{B}=3 \times 10^{4} \mathrm{~T} \mathrm{~m}^{-1}$ at the center of the tweezers. For poles that have a limited thickness, the gradient is reduced. The poles are fabricated by electroplating cobalt onto microscope coverslip glasses; a detailed description of this procedure has been published (de Vries et al. 2004). Cobalt is chosen because of its high magnetic saturation (1.8 T) (Maykuth 1979), its low remanence, and its stability in an aqueous environment. The fabricated poles have a tip radius of $\sim 3 \mu \mathrm{m}$ and a thickness of $5 \mu \mathrm{m}$. In order to estimate the performance of the magnetic tweezers (e.g. by numerical calculation) it is important to know the magnetic properties of the poles. These properties can be analyzed using a vibrating sample magnetometer (VSM). Measurement of the magnetic properties of the fabricated cobalt poles resulted in the following parameters: saturation magnetization $M_{\text {sat }}=1.1 \times 10^{3}$ $\mathrm{kA} \mathrm{m}{ }^{-1}$; coercive force $H_{\mathrm{c}}=9 \mathrm{kA} \mathrm{m}^{-1}$, and remanence $B_{\mathrm{r}}=5 \times 10^{2} \mathrm{kA} \mathrm{m}^{-1}$.

Before the magnetic tweezers are used for experiments it is important to generate a force calibration curve. This calibration establishes how the force on a magnetic particle depends on the current through the coils. In practice it is not adequate to use calculations, because of uncertainties in the exact geometry and magnetic parameters of the materials involved (yoke, poles). Furthermore, the magnetic moment of the particles used is in most cases not exactly specified. Different strategies for force calibration have been developed. The most frequently applied method is tracking of the displacement of a 
magnetic particle through stationary fluids of known viscosity. The external magnetic force on the particle is determined from $F=\gamma v$, with $v$ the terminal velocity of the particle. For spherical particles, the drag coefficient $\gamma$ is given by Stokes' law $\gamma=6 \pi \eta r$, where $r$ is the radius of the particle. This method works very well for single-pole magnetic tweezers but is less adequate in multipole geometries as used here because of the limited space in between the poles. Alternatively, cantilever-based calibration methods can be used. Here, the magnetic particle is glued to the tip of a cantilever with known spring constant $k$. By measuring the deflection $\Delta x$ of the cantilever, caused by the magnetic force that is exerted on the bead, the force is calculated as $F=k \Delta x$. We use this approach as it is ideally suited for measuring forces in our multipole MT designs. As a cantilever we use home-made micropipettes (de Vries et al. 2005) that are calibrated against an AFM cantilever with known spring constant (TM microscopes (Veeco, Woodbury, NY, USA), microlevers tip B, spring constant $10 \mathrm{nN} \mu \mathrm{m}^{-1}$ ). Typically, spring constants of the micropipettes were $0.8 \mathrm{nN} \mu \mathrm{m}^{-1}$. A superparamagnetic bead with well-defined magnetic properties (Dynabeads M280, Dynal, Oslo, Norway; volume magnetization $11.5 \mathrm{kA} \mathrm{m}^{-1}$ ) is glued (using two-component epoxy glue) to the end of the pipette. The assembly is positioned carefully such that the superparamagnetic bead is placed at the center of the magnetic tweezers. Deflection of the micropipette is recorded using video microscopy and analyzed off-line using Labview-based homemade particle tracking software. The method allows to measure forces down to $\sim 10 \mathrm{pN}$. An example of a measured force calibration curve is shown in Figure 3 which also depicts the calculated forces for comparison. The maximum force measured is $\sim 1 \mathrm{nN}$, corresponding to a gradient $\nabla \boldsymbol{B}=8 \times 10^{3} \mathrm{~T} \mathrm{~m}^{-1}$ at the center of the tweezers. It is now possible to predict the maximum forces that can be exerted on magnetic beads once their magnetic properties are known. An overview of the magnetic properties and corresponding forces are given for a selection of commercially available particles in Table 1. As is evident, the force drops rapidly with decreasing bead size. For comparison, the force of a $100 \mathrm{~nm}$ iron particle is also given. The calculated maximum achievable forces are important for the success of the application of magnetic tweezers for chromatin manipulation as will be discussed later.

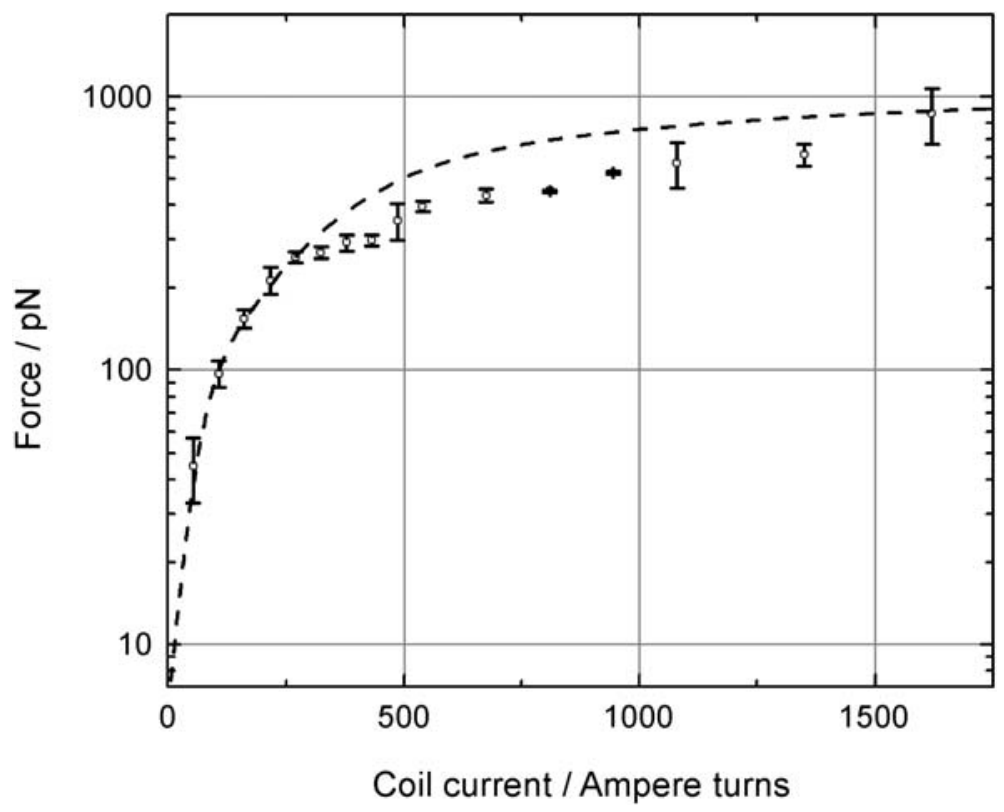

Figure 3. Measured (circles) and calculated (dashed line) forces exerted on a superparamagnetic bead (M280 from Dynal) positioned at the center of a three-pole magnetic tweezers plotted for different coil currents. Reproduced with permission from de Vries et al. (2005). Copyright 2005 the Biophysical Society. 
Table 1. Overview of the magnetic properties and corresponding maximum forces that can be exerted using the current MT set-up for a selection of magnetic beads

\begin{tabular}{lllr}
\hline Bead type & Diameter $(\mu \mathrm{m})$ & Volume magnetization $\left(\mathrm{kAm}^{-1}\right)$ & Maximum force $(\mathrm{pN})$ \\
\hline DynaBeads-M280 (Dynal) & 2.80 & 11.5 & 1000 \\
MyOne (Dynal) & 1.05 & 28.4 & 150 \\
$47 \% \gamma$-Fe $\mathrm{O}_{4}$ (Bangs Labs) & 0.35 & 65 & 12 \\
$100 \%$ iron beads & 0.10 & $1.7 \times 10^{3}$ & 7 \\
\hline
\end{tabular}

\section{Mechanical properties of chromatin in living cells}

The magnetic tweezers described in the previous section were successfully used to manipulate superparamagnetic beads inside the nucleus of a living cell. Following the trajectory of a micrometer-sized superparamagnetic bead in response to an external magnetic force allowed the determination of the local mechanical properties of the nuclear architecture. The first step in these experiments is to get a superparamagnetic bead inside the nucleus of a cell.
This is most conveniently done by microinjection. The challenge here is to inject a single bead inside the nucleus. Methods for microinjection like those used in microparticle tracking rely on the injection of a given volume of a suspension of beads or of a ferrofluid (Tseng et al. 2004). This method allows no control of the precise number of beads that are introduced in the nucleus. We therefore adapted this method for single-bead injection. To this end, a single superparamagnetic bead (MyOne, Dynal, $r=0.5 \mu \mathrm{m})$ is sucked onto the tip of a micropipette.

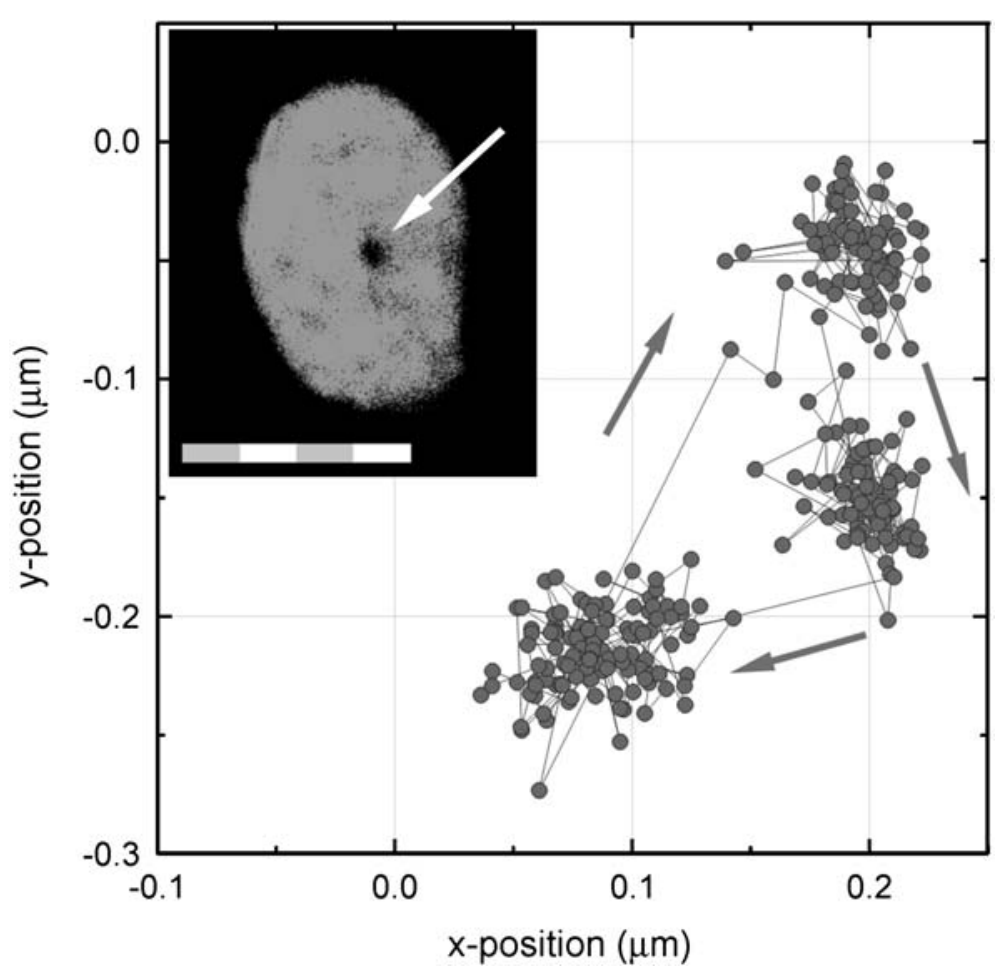

Figure 4. Measured trajectory of a superparamagnetic bead inside the nucleus of a living HeLa cell induced by a magnetic force of $110 \mathrm{pN}$ alternating clockwise between three different directions indicated by the arrows. A single triangular path is shown. The inset represents a confocal section through the nucleus of the HeLa cell showing the distribution of GFP-tagged histone H2B. The nucleus contains a single microinjected bead indicated by the arrow. Scale bar is $10 \mu \mathrm{m}$. Reproduced with permission from de Vries et al. (2007). Copyright 2007 the American Chemical Society. 
The micropipette has an inner diameter of $0.4 \mu \mathrm{m}$ (World Precision Instruments, Inc., Sarasota, Florida, USA, TIP04TW1F micropipette), which is smaller than the bead diameter. The pipette is connected to an Eppendorf Femtojet microinjector to control the pressure. Varying the compensation pressure $(\sim 5-15 \mathrm{kPa})$ causes an influx of medium into the pipette, allowing the capture of the bead onto the tip. HeLa cells, expressing green fluorescence protein (GFP)-tagged histone H2B uniformly labeling all the chromatin, were deposited on a glass coverslip and left for $2 \mathrm{~h}$ to attach and spread before proceeding with the microinjection. A micromanipulator is used to push the superparamagnetic bead into the nucleus of a HeLa cell. Next, the bead is released from the pipette by increasing the compensation pressure in the micropipette. Finally, the micropipette is withdrawn from the cell, leaving a single superparamagnetic bead inside the nucleus. Confocal fluorescence microscopy of the nucleus is used to confirm the successful microinjection procedure (see inset, Figure 4). The microinjection procedure showed a high success rate and did not cause any observable morphological changes (e.g. swelling or bleb formation) in the cells, indicating that the cells are not negatively affected by this procedure.

After successful injection of the bead into the nucleus of the cell, the superparamagnetic bead is subjected to external magnetic forces using the threepole magnetic tweezer set-up as described above. Manipulation experiments were performed for two different amplitudes of the magnetic force $(110 \mathrm{pN}$ and $65 \mathrm{pN}$ ). The direction of the force was always towards one of the poles, and was changed stepwise from pole 1 to pole 2 , to pole 3 , back to pole 1 , and so on in a clockwise manner. Simultaneously, bead movements were captured on video and stored for later analysis. Bead positions were determined by offline video analysis. An example of a bead trajectory for a full cycle is shown in Figure 4. Clearly, a triangular path is described showing a fast elastic response (the arms of the triangle) when the force changes direction, after which the bead seems to settle around an equilibrium position (the corners of the triangle). The 'clouds' of data points that are visible at the corners of the triangles are due to 'noise' caused by the limited accuracy in determining the bead position. It appeared not to be possible to move the bead over longer distances, indicating that the nuclear architecture behaves as a highly viscous medium for bead sizes of $1 \mu \mathrm{m}$. This proofof-principle experiment convincingly shows that controlled movement of magnetic beads inside the nucleus of a cell is feasible. In addition, detailed analysis of the data obtained from this particular experiment provides useful information on the nuclear architecture.

Micromechanical properties derived from the bead displacement data can be used to validate existing models or to develop new models of nuclear architecture. The analysis starts with plotting the normalized bead displacement versus time (Figure 5). These data are extracted from the measured bead trajectories by calculating the displacements of the bead and normalizing with the applied force. The displacements are determined with respect to the mean position of the previous 'corner'. The data in Figure 5 are obtained after averaging nine individual traces for a single cell. The response function thus obtained clearly describes an environment that is viscoelastic. A fast elastic response is followed by a viscous behavior for longer time scales. Analytically this behavior is best described by a so-called Voigt-Maxwell body (Figure 5, inset), which consists of a series system of a viscoelastic body (a dashpot characterized by damping coefficient $\gamma_{1}$ in parallel with a spring constant $k$ ) with a dashpot characterized by damping coefficient $\gamma_{0}$ (Feneberg et al. 2001). In this case the normalized displacement of the bead evoked by a stepwise force $F$ is expressed as

$\frac{s(t)}{F}=\frac{1}{k}\left[1-\exp \left(-\frac{t}{\tau}\right)\right]+\frac{t}{\gamma_{0}}$

with the relaxation time $\tau$ given by

$\tau=\frac{\gamma_{1}}{k}$

In this model, $k$ is a measure of the spring constant of the structure and describes the fast initial response of the bead upon the application of an external force. The damping coefficient $\gamma_{1}$ determines the speed at which the bead is displaced during the initial response. The relaxation time $\tau$ as defined in equation (1b) describes the time required for the transition from the elastic regime to the viscous flow regime. Finally, $\gamma_{0}$ describes the effective viscous drag coefficient of the bead in the viscous flow regime at longer time scales. Curve fitting of the data using equations $1 \mathrm{a}$ and $1 \mathrm{~b}$ yields the following values for 


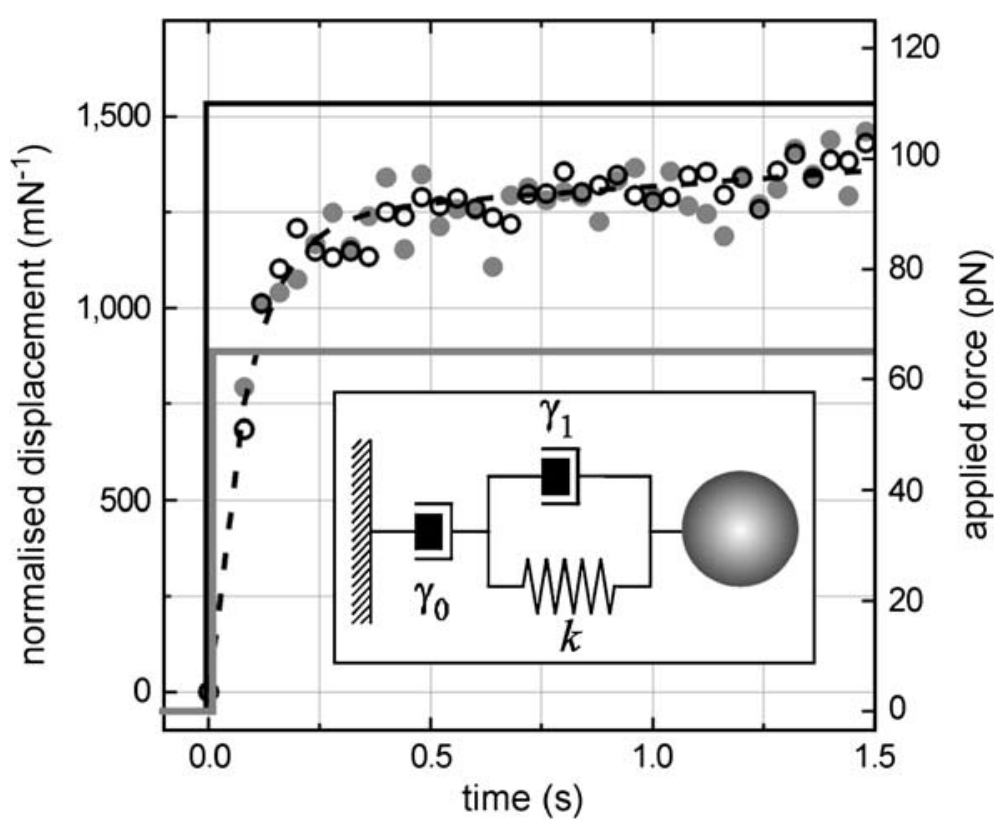

Figure 5. Measured response function, normalized by force (left axis) of a bead in the nucleus after application of $65 \mathrm{pN}$ (solid circles) and $110 \mathrm{pN}$ (open circles) force steps (solid lines, right axis). The dashed line is a curve fitted using a viscoelastic model, characterized by damping coefficients $\gamma_{0}$ and $\gamma_{1}$ and by a spring constant $k$, as shown in the inset. Reproduced with permission from de Vries et al. (2007). Copyright 2007 the American Chemical Society.

the fit parameters $k, \gamma_{0}$ and $\gamma_{1}: k=8.0 \times 10^{-4} \mathrm{~Pa} \mathrm{~m}$; $\gamma_{0}=1.2 \times 10^{-2} \mathrm{~Pa} \mathrm{~s} \mathrm{~m} ; \gamma_{1}=7.0 \times 10^{-5} \mathrm{~Pa}$ s m. These parameters relate to the viscoelastic properties of the cell nucleus. Following Stokes' law, the viscosity $\eta$ is given by $\eta=\gamma / 6 \pi r$, where $r$ is the radius of the magnetic bead. The Young's modulus $Y$ is expressed as $Y=3 \mu$, where $\mu$ is the shear modulus (Landau 1959) and is given by $\mu=k / 6 \pi r$ (Ziemann et al. 1994). This results in a Young's modulus $Y=250 \mathrm{~Pa}$ and a viscosity $\eta_{0}=1.2 \times 10^{3} \mathrm{~Pa}$ s. The fitting errors are $\sim 10 \%$. The observed values vary slightly (50\%), however, depending on the direction of the bead displacements and from cell to cell.

The observed almost purely elastic behavior of the nuclear material is consistent with previous observations using cell poking (Heidemann et al. 1999) and AFM measurements (Mathur et al. 2000). However, the elasticity found here is about 10 - to 100 -fold lower than what was reported by micropipette and AFM methods (Lammerding et al. 2007). The main difference between these techniques and the MT method is that with micropipette and AFM the elasticity is determined by poking the nucleus from the outside. Therefore, the elasticity measured arises from both the nuclear envelope and the interior of the nucleus. However, with MT the elasticity is determined of the interior of the nucleus only. This may explain the observed lower values of stiffness found here compared with the micropipette and AFM methods. In addition, different cell types are used, which can also give rise to different mechanical properties. Comparison of the observed Young's modulus with values obtained by micropipette experiments on isolated mitotic chromosomes shows very good agreement. These studies (Marko \& Poirier 2003) show an elastic modulus of typically $500 \mathrm{~Pa}$, a value only slightly larger than the $250 \mathrm{~Pa}$ observed in the MT studies. Because the force range and the length-scale that are probed are quite similar for both methods, these results suggest that, although the internal structure might be different, mitotic chromosomes and interphase chromatin have similar elastic properties. The values obtained here may best be compared to a study that used PTM (Tseng et al. 2004). The viscosity was found to be about $500 \mathrm{~Pa} \mathrm{~s}$, a value very close to our observations. The average 
elasticity is reported to be about $18 \mathrm{~Pa}$, which is 10-fold lower than observed with the MT method. However, the observed high viscosity and elasticity are in contrast with data obtained with FRAP and fluorescence correlation microscopy (FCS) applied, for example, to fluorescently labeled nuclear proteins and polymers. Those studies show a nuclear viscosity that is orders of magnitude smaller and resembles that of water (Lukacs et al. 2000, Pederson 2000, Verschure et al. 2003). This discrepancy can be explained by realizing that, by using the relatively small nuclear proteins, one probes predominantly the interstitial viscosity of the liquid phase of the nucleus, whereas using the magnetic beads, which are much larger than the effective mesh size of the intranuclear region, one probes the mechanical properties of the nucleus at the meso-scale including chromatin and nuclear bodies (Tseng et al. 2004).

The general view of chromatin structure in interphase nuclei is one in which the $10 \mathrm{~nm}$ nucleosomal chain is folded into a helical structure called the $30 \mathrm{~nm}$ fiber, which in turn is organized in a so far unknown higher-order structure (Adkins et al. 2004). The $30 \mathrm{~nm}$ fiber can be treated as a flexible polymer. One approach, in which the chromatin is described as a crosslinked polymer network, correctly predicts the observed Young's modulus (see de Vries et al. 2007 for a detailed description). Here we only highlight the main aspects of the model. The Young's modulus of a crosslinked polymer network is given by (Doi 1986)

$Y=6 n k_{B} T$

where $k_{\mathrm{B}}=1.3 \times 10^{-23} \mathrm{~J} / \mathrm{K}$ is Boltzmann's constant and $T$ is the absolute temperature. On average the distance between crosslinks can never exceed the length of the fiber between two crosslinks. Because the distance between crosslinks is determined by the crosslinker density $n$, the value of $n$ is limited to

$n \leq(L / 2 \Theta V)^{3 / 2}$

where $\Theta$ represents the part of the nucleus that is taken up by the sum of all chromosome territories, and ranges from 0 to $1, \Theta=1$ represents the situation in which chromatin occupies the total nuclear volume $V$. The total length of chromatin fiber is given by $L$. Evaluation of the Young's modulus using equations (2) and (3) (with $V=315 \mu \mathrm{m}^{3} ; L=5.5 \times 10^{-2} \mathrm{~m}$ ) for different values of the crosslinker density $n$ and volume fraction $\Theta$ is shown in Figure 6. From this figure it is concluded that a volume fraction of $\Theta=0.19$ yields a maximum value of the Young's modulus of $Y \leq 250 \mathrm{~Pa}$, corresponding to the exper-

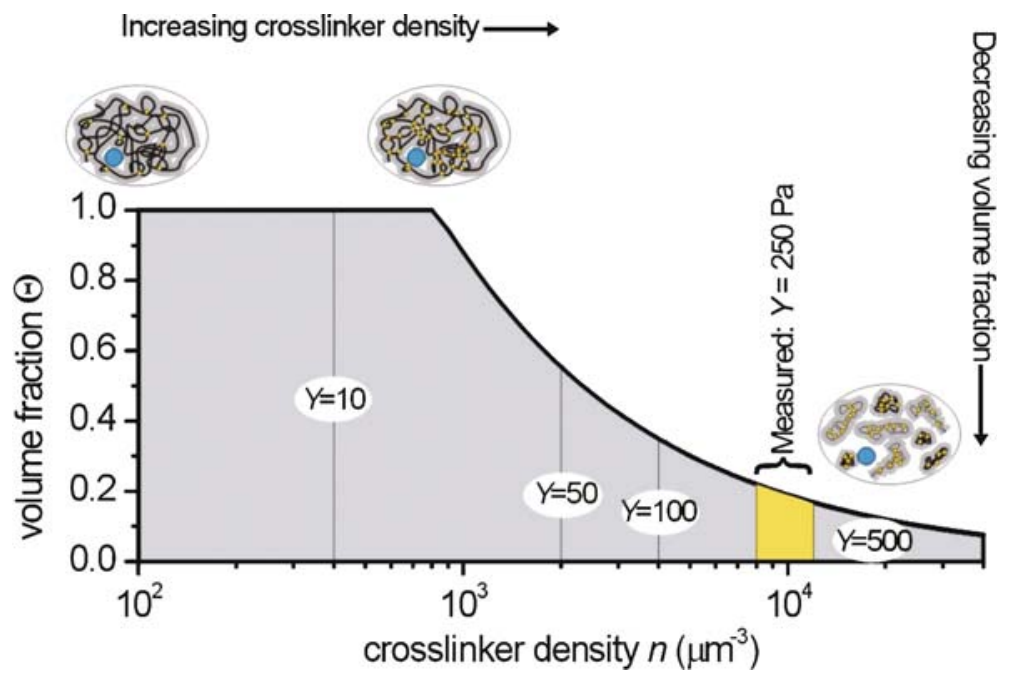

Figure 6. Interpretation of nuclear architecture based on a polymer network model of chromatin. Vertical solid lines indicate possible combinations of volume fraction and crosslinker density to yield the specified Young's modulus. The measured Young's modulus of $250 \mathrm{~Pa}$ corresponds to a $\theta<1$, in agreement with the general picture of chromatin organization in compact domains. 
imental results. In this case the length of the fiber between two crosslinks is also reduced and approaches the value of the persistence length. However, the polymer network model is only valid if the length of the fiber between two crosslinks is much larger than the persistence length. Therefore, the value of $\Theta$ obtained can be regarded as a lower limit. More advanced modeling is required to correctly include the persistence length of the fiber and to include other factors such as the stiffness of the crosslinks. We continue to develop these ideas in ongoing work. The current model suggests a volume fraction $\Theta$ that is considerably smaller than unity, a prediction that is in agreement with current understanding of interphase chromatin organization.

No precise measurements have been made of the nuclear fraction $\Theta$. Electron-microscopic images of thin sections of nuclei suggest that this value does not exceed 0.5 and might be considerably smaller, depending on the cell type (Cmarko et al. 2003). We speculate that the molecular basis of the crosslinking of the chromatin fiber inside the chromosome territory may be related to the putative arrangement of chromatin in loops (Heng et al. 2004). Alternatively, crosslinks between nucleosomes may occur via chromatin-associated proteins, such as HP1, which binds to histone $\mathrm{H} 3$ methylated at lysine 9 and can form homodimers (Eissenberg \& Elgin 2000). However, other chromatin proteins may also be involved, such as the methylated DNA-binding protein MeCP2 (Georgel et al. 2003).

\section{Discussion and future directions}

As demonstrated, the controlled manipulation of small beads inside the nucleus of a living cell is feasible and can provide important and indeed novel information on the mechanical properties of chromatin structure. The in vivo elasticity and viscosity observed are in reasonable agreement with those obtained with PTM and allow estimation of the forces that are required, for example, to reposition (parts of) chromatin within the nucleus. If only the elasticity is considered, a force of about $100 \mathrm{pN}$ on a $1 \mu \mathrm{m}$ bead caused a bead displacement of about $100 \mathrm{~nm}$. As a result, the local chromatin is also repositioned over the same distance. Larger displacements will require larger forces or alternatively smaller beads. Larger forces can readily be obtained by using magnetic particles with higher volume magnetizations. In this way the use of iron particles can increase the maximum force by a factor of 60 . Therefore, it is anticipated that displacements on the order of micrometers are feasible. The use of smaller beads is also possible. Although the exerted forces are considerable lower (e.g. $7 \mathrm{pN}$ for a $100 \mathrm{~nm}$ iron particle with the current MT set-up), the observed Young's modulus for $100 \mathrm{~nm}$ particles is also lower by an order of magnitude (Tseng et al. 2004). This results in an estimated maximum displacement in the micrometer range for a $100 \mathrm{~nm}$ particle, comparable to the maximum displacement of a micrometer-sized particle. These estimated displacements, which are

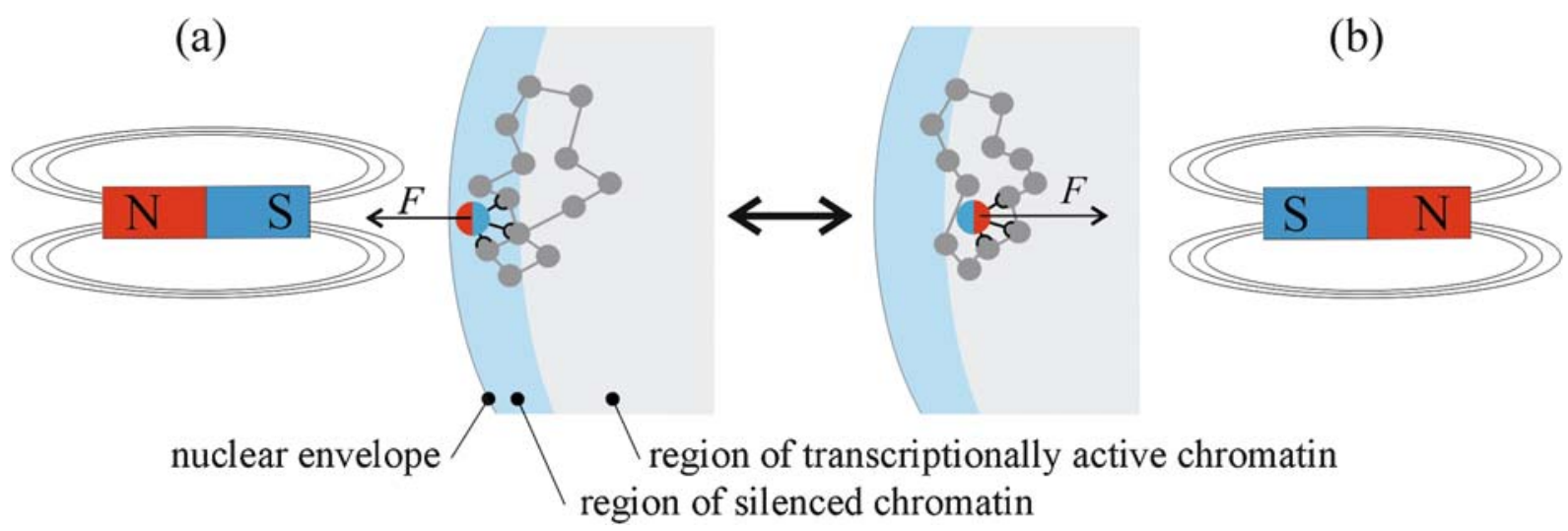

Figure 7. Using magnetic tweezers to reposition loci that are bound to a magnetic nanobead from (a) a region of silenced chromatin close to the periphery of the nucleus towards (b) a region of transcriptionally active chromatin. Adapted from Mateos-Langerak et al. (2007). 
based on the observed elasticity, take place on a short time scale (seconds). The long-term behavior of the particle inside the nucleus is governed by the viscosity. As discussed for very small particles (like proteins), the viscosity is very low and large macromolecules of many kilodaltons can easily diffuse throughout the nucleus and into chromatin domains (Verschure et al. 2003). However, for particles with a size of $100 \mathrm{~nm}$ the viscosity is already many orders of magnitude larger and therefore strongly hampers the relocation of these particles. More experiments are required with particles much smaller than the $1 \mu \mathrm{m}$ particles used so far to get conclusive answers on the possibility of maneuvering small magnetic beads through the nucleus and chromatin domains to any desired position.

Based on the analysis above, we believe that several interesting experiments are feasible using the current MT method. One of them is the in situ manipulation of nuclear organization (Mateos-Langerak et al. 2007). The transcriptional activity of a gene is related to its local environment in the nucleus (Goetze et al. 2007). This suggests that changing the position of a genomic locus in the nucleus while simultaneously observing the effect on transcription and replication is a possible approach to analyzing the relationship between nuclear position and activity of a gene. Manipulation of magnetic particles in the nucleus may be a promising candidate for repositioning loci. To this end, magnetic particles, coated with, for instance, antibodies against core histones, could be injected into the nucleus close to the nuclear periphery. MT could then be used to displace the particle and the bound loci towards the center of the nucleus. The estimated micrometer displacements may be large enough to reposition the loci from a zone of silenced chromatin to a zone of transcriptionally active chromatin (Figure 7).

Other applications may focus on inducing local chemical modifications to chromatin structure. As an example, magnetic particles could be coated with enzymes that induce methylation or de-methylation of histone tails and in this way modify the local chromatin structure to allow study of its effect on gene activity.

The first experimental results and the unique ability to interfere with chromatin structure lend credence to the use of MT for the study of nuclear architecture. The method is fully compatible with optical microscopy techniques, such as confocal fluorescence microscopy. However, there are some potential limitations to this technique. First, as discussed, the viscosity is very high for large beads, strongly hampering the ability to maneuver the particles through the nucleus. The use of much smaller beads will probably solve this issue but at the cost of much lower magnetic forces. Secondly, a so far unaddressed issue is the possible nonspecific interaction of the bead with nuclear components such as proteins, etc. Investigating the existence of these nonspecific interactions, quantifying their effects, and ultimately reducing them (e.g. by coating the beads with polyethyleneglycol) will form key components of the refinement of the MT manipulation technique in the future.

\section{Conclusions}

In this paper we review and discuss the possibilities for manipulating chromatin inside live cells using magnetic particles. As a 'minimally invasive' technique, MT has unique advantages that allow experimental studies on chromatin structure that are not easily accomplished otherwise. We show that the MT method has many feasible applications for the study of structure-function relationships of chromatin. But it should also be realized that this technology has only just begun to develop and more work is required to fully explore the potential of this method for the study of nuclear architecture. Finally, we believe that the work presented here opens new avenues of research for structure-function correlation of mechanical properties in the most challenging and complex cellular milieu.

\section{References}

Adkins NL, Watts M, Georgel PT (2004) To the 30-nm chromatin fiber and beyond. Biochim Biophys Acta 1677: 12-23.

Bausch AR, Ziemann F, Boulbitch AA, Jacobson A, Sackmann E (1998) Local measurements of viscoelastic parameters of adherent cell surfaces by magnetic bead microrheometry. Biophys J 75: 2038-2049.

Bausch AR, Moller W, Sackmann E (1999) Measurement of local viscoelasticity and forces in living cells by magnetic tweezers. Biophys J 76: 573-579.

Bennink ML, Leuba SH, Leno GH, Zlatanova J, de Grooth BG, Greve J (2001) Unfolding individual nucleosomes by stretching single chromatin fibers with optical tweezers. Nat Struct Biol 8: 606-610. 
Caspi A, Granek R, Elbaum M (2002) Diffusion and directed motion in cellular transport. Phys Rev E 66: 1-12.

Cmarko D, Verschure PJ, Otte AR, van Driel R, Fakan S (2003) Polycomb group gene silencing proteins are concentrated in the perichromatin compartment of the mammalian nucleus. $J$ Cell Sci 116: 335-343.

Crick FHC, Hughes AFW (1950) The physical properties of cytoplasm: a study by means of the magnetic particle method part I. experimental. Exp Cell Res 1: 37-80.

Cui Y, Bustamante C (2000) Pulling a single chromatin fiber reveals the forces that maintain its higher-order structure. Proc Natl Acad Sci U S A 97: 127-132.

Dahl KN, Engler AJ, Pajerowski JD, Discher DE (2005) Powerlaw rheology of isolated nuclei with deformation mapping of nuclear structures. Biophys J 89: 2855-2864.

de Vries AHB, Kanger JS, Krenn BE, van Driel R (2004) Patterned electroplating of micrometer scale magnetic structures on glass substrates. J Microelectromech S 13: 391-395.

de Vries AHB, Krenn BE, van Driel R, Kanger JS (2005) Micro magnetic tweezers for nanomanipulation inside live cells. Biophys $J$ 88: 2137-2144.

de Vries AHB, Krenn BE, van Driel R, Subramaniam V, Kanger JS (2007) Direct observation of nanomechanical properties of chromatin in living cells. Nano Lett 7: 1424-1427.

Doi, M (1986) Introduction to Polymer Physics. Oxford: Clarendon Press.

Eissenberg JC, Elgin SC (2000) The HP1 protein family: getting a grip on chromatin. Curr Opin Genet Dev 10: 204-210.

Feneberg W, Westphal M, Sackmann E (2001) Dictyostelium cells' cytoplasm as an active viscoplastic body. Eur Biophys $J$ 30: 284-294.

Fisher JK, Cummings JR, Desai KV et al. (2005) Threedimensional force microscope: a nanometric optical tracking and magnetic manipulation system for the biomedical sciences. Rev Sci Instrum 76: 053711/1-053711/11.

Georgel PT, Horowitz-Scherer RA, Adkins N, Woodcock CL, Wade PA, Hansen JC (2003) Chromatin compaction by human MeCP2. Assembly of novel secondary chromatin structures in the absence of DNA methylation. J Biol Chem 278: 32181-32188.

Goetze S, Mateos-Langerak J, Gierman HJ et al. (2007) The three dimensional structure of human interphase chromosomes is related to the transcriptome map. Mol Cell Biol 27: 4475-4487.

Heidemann SR, Kaech S, Buxbaum RE, Matus A (1999) Direct observation of the mechanical behaviors of the cytoskeleton in living fibroblasts. J Cell Biol 145: 109-122.

Heng HHQ, Goetze S, Ye CJ et al. (2004) Chromatin loops are selectively anchored using scaffold/matrix attachment regions. J Cell Sci 117: 999-1008.

Jeffries GDM, Edgar JS, Zhao Y, Shelby JP, Fong C, Chiu DT (2007) Using polarization-shaped optical vortex traps for single cell nanosurgery. Nanoletters 7: 415-420.

Lammerding J, Dahl KN, Discher DE, Kamm RD (2007) Nuclear mechanics and methods. Methods Cell Biol 83: 269-294.
Landau L, Lifchitz E (1959) Theory of Elasticity. Oxford: Pergamon Press

Lukacs GL, Haggie P, Seksek O, Lechardeur D, Freedman N, Verkman AS (2000) Size-dependent DNA mobility in cytoplasm and nucleus. J Biol Chem 275: 1625-1629.

Marko JF, Poirier MG (2003) Micromechanics of chromatin and chromosomes. Biochem Cell Biol 81: 209-220.

Mateos-Langerak J, Goetze S, Leonhardt $\mathrm{H}$, Cremer $\mathrm{T}$, van Driel R, Lanctot C (2007) Nuclear architecture: is it important for genome function and can we prove it? $J$ Cell Biochem 102(5): 1067-1075.

Mathur AB, Truskey GA, Reichert WM (2000) Atomic force and total internal reflection fluorescence microscopy for the study of force transmission in endothelial cells. Biophys $J \mathbf{7 8}$ : 1725-1735.

Maykuth DJ, ed. (1979) Cobalt. Metals Handbook, 9th edn, vol. 2: 725-726.

Obataya I, Nakamura C, Han S, Nakamura N, Miyake J (2005a) Mechanical sensing of the penetration of various nanoneedles into a living cell using atomic force microscopy. Biosens Bioelectron 20: 1652-1655.

Obataya I, Nakamura C, Han S, Nakamura N, Miyake J (2005b) Nanoscale operation of a living cell using an atomic force microscope with a nanoneedle. Nanoletters 5: 27-30.

Pederson T (2000) Diffusional protein transport within the nucleus: a message in the medium. Nat Cell Biol 2: E73-E74.

Phair RD, Misteli T (2000) High mobility of proteins in the mammalian cell nucleus. Nature 404: 604-609.

Shelby JP, Edgar JS, Chiu DT (2005) Monitoring cell survival after extraction of a single subcellular organelle using optical trapping and pulsed-nitrogen laser ablation. Photochem Photobiol 81: 994-1001.

Skibbens RV, Salmon ED (1997) Micromanipulation of chromosomes in mitotic vertebrate tissue cells: tension controls the state of kinetochore movement. Exp Cell Res 235: 314-324.

Strick TR, Allemand JF, Bensimon D, Bensimon A, Croquette V (1996) The elasticity of a single supercoiled DNA molecule. Science 271: 1835-1837.

Thoumine O, Ott A (1997) Time scale dependent viscoelastic and contractile regimes in fibroblasts probed by microplate manipulation. J Cell Sci 110: 2109-2116.

Tseng Y, Lee JSH, Kole TP, Jiang I, Wirtz D (2004) Microorganization and visco-elasticity of the interphase nucleus revealed by particle nanotracking. J Cell Sci 117: 2159-2167.

Verschure PJ, van der Kraan I, Manders EM, Hoogstraten D, Houtsmuller AB, van Driel R (2003) Condensed chromatin domains in the mammalian nucleus are accessible to large macromolecules. EMBO Reports 4: 861-866.

Ziemann F, Radler J, Sackmann E (1994) Local measurement of viscoelastic moduli of entangled actin networks using an oscillating magnetic bead micro-rheometer. Biophys $J$ 66: 2210-2216. 